\title{
Setback for JAK2 inhibitors
}

The US Food and Drug Administration put a clinical hold on all trials of Sanofi's fedratinib, a highly selective JAK2 inhibitor, on November 13. Less than a week later, the Paris-based big pharma pulled the plug on the program. The agency's hold came after patients in studies of the molecule developed symptoms consistent with Wernicke's encephalopathy, a condition caused by brain lesions resulting from an inadequate supply of thiamine. But competitors say the problem is molecule specific and are shrugging off any impact on their programs.

Fedratinib is a small-molecule inhibitor of Janus kinase 2 (JAK2), which Sanofi obtained through its \$75-million acquisition of San Diego-based TargeGen in 2010. JAK family proteins help orchestrate the immune response by enabling cytokines

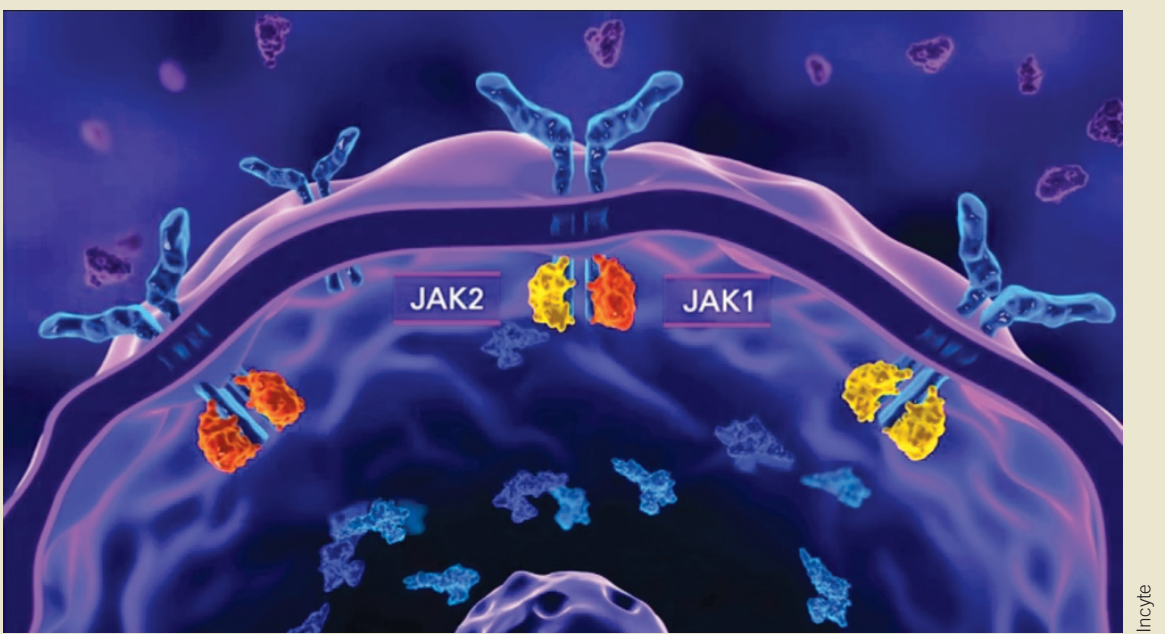

Incyte's Jakafi approval for myelofibrosis validated the role of Janus-kinases JAK1 and JAK2 in cancer.

to signal through their receptors. That makes their inhibition tricky business, and developers of JAK inhibitors have been acutely aware of the potential for long-term side effects. Fedratinib was in development for the treatment of myeloproliferative disorders and had advanced to a phase 3 study in myelofibrosis, a rare, lifethreatening blood cancer, which was to have been the basis for filing for approval. "As we do not know what are the underlying cause(s) of certain patients being at higher risk for this adverse event, there is currently no patient selection strategy that would mitigate this risk," a Sanofi spokesperson said in an e-mail.

Incyte's Jakafi (ruxolitinib), a JAK1/JAK2 inhibitor, became the first drug approved to treat myelofibrosis, in 2012 (Nat. Biotechnol. 30, 3, 2012). New York-based Pfizer's JAK3-inhibiting Xeljanz (tofacitinib), approved in November 2012 for rheumatoid arthritis, is the only other JAK inhibitor on the market (Nat. Biotechnol. 31, 3, 2013).

Incyte, which has additional JAK inhibitors in development for oncologic and inflammatory conditions, has been following the fedratinib situation closely. Richard Levy, chief development and medical officer at the Wilmington, Delaware-based biotech, says the first results of an in-house program to try and elucidate the mechanism of why fedratinib was causing Wernicke's encephalopathy indicate that it is not a class effect of JAK inhibitors. "We work in a very different structural space in terms of how we inhibit JAKs," he says. Thus, he does not believe there's any concern that a yet-unidentified subgroup of myelofibrosis patients could be at risk. "It's not about the patient, it's about the molecule," he says. Incyte expects to submit the results of its structural analysis of different JAK inhibitors for publication in the next month or two.

Several JAK-targeting compounds have fallen by the wayside during clinical development, including London-based AstraZeneca's AZD1480; lestaurtinib from Cephalon (now part of Teva

Pharmaceutical in Petach Tikva); and S. San Francisco-based Exelixis' XL014. Of those left standing, Gilead Sciences' JAK1/JAK2 momelotinib (obtained through the Foster City, California, company's acquisition of YM Biosciences in 2012) is in a head-to-head phase 3 myelofibrosis study versus Jakafi. Seattle-based Cell Therapeutics has pacritinib in phase 3 as well. It acquired the dual inhibitor of JAK2 and fms-related tyrosine kinase 3 (FLT3) from Singapore's S*BIO in 2012 after S. San Francisco's Onyx Pharmaceuticals, which had an option on the molecule from $\mathrm{S} * \mathrm{BIO}$ and had provided over $\$ 100$ million in development support, declined to do so.

Cell Therapeutics' CEO James Bianco also says that the fedratinib situation is unique to that molecule. Structural analysis of Jakafi and pacritinib, he says, show they are distinct from the rest. They cannot form the linear shape that is consistent with the thiamine structural configuration and with competitive inhibition of thiamine uptake and metabolism. "All the rest can," he says, including fedratinib, AZD1480 and momelotinib.

Mark Ratner Cambridge, Massachusetts

\section{IN brief}

\section{UK boosts discovery tools}

In November, the UK's Medical Research Council (MRC) announced funding up to $£ 150$ (\$246) million for projects aimed at strengthening the UK's capacity to explore new avenues in clinical research. The focus is on improving research networks and infrastructure in three specific areas: stratified and experimental medicine, single-cell functional genomics and dementia research. "We want to build up a national network [in clinical research] across universities and other research institutions so that people are working in a way that is complementary," says Anne Marie Coriat, director of capacity, skills and infrastructure at MRC. Consequently, proposals that involve multiple institutions or industry will be favored. As an example of how strengthening infrastructure can advance science, Coriat points to MRC's funding of health informatics infrastructure, which has enabled researchers in England, Scotland and Wales to link patient clinical records with other forms of routinely collected environmental, social and economic data to conduct public health studies. Infrastructure could include methods for ex vivo sample analysis, remote sensing of physiological functions and real-time visualization of biomedical processes in disease, and methods that integrate state-of-the-art technologies with clinical research. "We need to invest to make sure the UK has got these technologies in the right place at the right time," Coriat adds. The MRC will administer the funds in collaboration with the UK Department of Health, Wellcome Trust, Cancer Research UK, the British Heart Foundation and Alzheimer's Research UK.

Gunjan Sinha 